\title{
Assessment of Yield and Economic for Kalmegh (Andrographis paniculata Nees) by Using Different Weed Control Techniques
}

\author{
Bolta Ram Meena $^{1 *}$, Shiv Singh Meena ${ }^{2}$ and D.C. Kala ${ }^{1}$ \\ ${ }^{1}$ Department of Agronomy, ${ }^{2}$ Department of Soil Science, College of Agriculture GBPUA \& T, \\ Pantnagar, Uttarakhand, India \\ *Corresponding author
}

\section{A B S T R A C T}

Keywords

Aromatic,

Randomized,

Replication,

Acanthaceae and Weedy.

Article Info

Accepted:

25 April 2017

Available Online:

10 May 2017
Medicinal and Aromatic plants form a numerically large group of economically important plants which provide basic raw materials for medicines, perfumes, flavors and cosmetics. These plants and their products not only serve as valuable source of income for small holders and entrepreneurs but also help the country to earn valuable foreign exchange by way of export. Kalmegh is an important medicinal plant that has been effectively used in traditional Asian medicines for centuries. A field experiment was conducted during kharif season of 2015 at G.B. Pant University of Agriculture and Technology, Pantnagar (Uttarakhand). The soil of the experimental area was silty clay loam in texture, the experiment, comprising of ten treatments, which were conducted in Randomized Block Design with three replications. All the treatments significantly increased fresh and dry herbage yield as compared to weedy check treatment. Maximum fresh and dry herbage yield $(25.070 \mathrm{t} / \mathrm{ha})$ was recorded in weed free treatment. The highest and lowest gross return, net return and $\mathrm{B}$ : $\mathrm{C}$ ratio was recorded in weed free and weedy check treatment, respectively.

\section{Introduction}

Medicinal plant is an integral part of human life to combat the sufferings from the dawn of civilization (Choudhary et al., 2010). It is estimated that more than 80,000 of total plant species have been identified and used as medicinal plants around the world (Joy et al., 1989). Andrographis paniculata is an important medicinal plant and widely used around the world. It belongs to the family Acanthaceae. Andrographis paniculata is used as a traditional herbal medicine in Bangladesh, China, Hong Kong, India, Pakistan, Philippines, Malaysia, Indonesia and Thailand and is ethnobotanically used for the treatment of snake bite, bug bite, diabetes, dysentery, fever and malaria. The genus Andrographis consists of 40 species and about 19 species are reported to be available in India, out of which Andrographis paniculata and Andrographis alata have medicinal properties. In India, kalmegh is cultivated as rainy season crop. The climatic requirement of the plant is hot and humid condition with ample sunshine. Kalmegh can be cultivated in wide range of soil from loam to lateritic soil with moderate fertility. 
All plant parts of kalmegh contain andrographolide but the leaves of kalmegh contain the highest amount of andrographolide. Kalmegh is a short duration crop and grown for medicinal purpose in kharif season and thus weed infestation is very high in this crop. Weeds deteriorate the quality and quantity of kalmegh, so weed management is very essential for maintaining the herbage yield and quality of plant. Hand weeding is best method of weed controlled if availability of labour Mirjha et al., (2013).

\section{Materials and Methods}

\section{Experimental site}

The experiment was conducted at the Medicinal Plants Research andDevelopment Centre, GB Pant University of Agriculture and Technology, U.S. Nagar (Uttarakhand) during the kharif season. The research centre lies in the taraibelt, $30 \mathrm{~km}$ southern end of foothills of Shivalik range of Himalaya at 290 latitude $79.50 \mathrm{E}$ longitude and at an altitude of $243.83 \mathrm{~m}$ above mean sea level. Tarai belt is characterized by a sub-tropical and sub humid climate in which summer is hot and dry and winter is severe cold. Winter season falls between October-February, then summer season continues till the end of June.

During summer, maximum temperature exceeds $400 \mathrm{C}$ while in winter, the minimum temperature occasionally touches 20C. Monsoon sets in the second or third week of June and continues up to the end of September. Some rain is also received during winter months. The average rainfall of Pantnagar is $1420 \mathrm{~mm}$ and most of rains are received from South-West monsoon from June to September. The soil of the experimental area was silty clay loam in texture being low in available nitrogen (215.76 kg ha-1), high in available phosphorus $(29.38 \mathrm{~kg}$ ha-1), medium in available potassium $(231 \mathrm{~kg}$ ha-1) and high organic carbon $(0.88 \%)$ contents with near neutral in reaction $(\mathrm{pH} 7.3)$.

\section{Experimental design and details of treatments}

The crop was transplanted on July 15, 2013 as per the randomized complete block design with three replications. The crop was transplanted in row $40 \mathrm{~cm}$ apart and $30 \mathrm{~cm}$ with in rows. Well rooted plants of $A$. paniculata cv. 'CIM-Megha' of uniform size and 45 days stage were transplanted in the experimental field followed by a light irrigation. The experiment, comprising of ten treatments (Table 1) viz., T1(Pendimethalin PE @ 1 kga.i. ha-1followed by mechanical weeding by hand hoe at 30-35 DAT), T2 (Quizalofop ethyl PoE at 3-5 leaf stage of weeds@50ga.i. ha-1followedby mechanical weeding by hand hoe at 30-35 DAT), T3 (Pendimethalin PE @ 1 kga.i. ha-1 + Quizalofop ethyl PoE at 3-5 leaf stage of weeds@ @ 50 ga.i. ha-1), T4 (Pendimethalin PE @ 1 kga.i. ha-1+ Quizalofop ethyl PoE at 3-5 leaf stage of weeds @ $50 \mathrm{~g}$ a.i. ha1 followed by mechanical weeding by hand hoe at 30-35 DAT), T5 (Pendimethalin PE @ $1 \mathrm{~kg}$ a.i. ha-1+ straw mulch @ 3 t ha-1), T6 (Pendimethalin PE @ 1kga.i. ha-1 + straw mulch@5 t ha-1), T7 (Two hand weeding at 15-20 and 30-35 DAT),T8 (Three hand weeding at 15-20, 30-35 and 45-50 DAT), T9 (Weedy check) and T10(Weed free).

\section{Observations}

The crop was harvested at 90 days after transplanting orat initiation of flowering, net plot of every treatment was harvested separately with help of sickle and kept in threshing floor under shade for drying the herb after taking the fresh weight of harvested every net plot. After complete drying of plants, dry weight was also taken to calculate dry herbage yield of net plot and accordingly yield (t/ha). 
Cost of cultivation was worked out treatment wise. The cost of labourers and mechanical power for various field operations (ploughing, harrowing, planking, transplanting, manual weeding, spraying and harvesting) was taken as per rate prevalent at the experimental farm. Cost of other inputs i.e., seeds, fertilizers and herbicides were calculated based on actual amount applied on hectare basis.

Gross return was obtained by dry matter yield with their respective market price. Net return was calculated by subtracting cost of cultivation from gross return. Benefit: cost ratio was calculated by dividing cost of cultivation from net return to assess the economics of kalmegh due to various treatments.

\section{Results and Discussion}

\section{Dry and fresh herbage yield}

The data recorded on dry and fresh herbage yield at 90 DAT (harvest) are presented in Table 3.1, Fig. 3.1. All the treatments significantly increased fresh herbage yield as compared to weedy check treatment Chandraker and Paikra (2015). Maximum fresh herbage yield $(25.070 \mathrm{t} / \mathrm{ha})$ was recorded in weed free treatment which was significantly higher than all other treatments, except T8 (Three hand weeding) treatment which was statistically at par similar result were observed by Bonasia et al., (2012).

The lowest fresh herbage yield (7.547 t/ha) was recorded in weedy check treatment which was significantly lower than all other treatments, except T3 (Pendi. PE @ 1 kg a.i./ha + Quizalofop ethyl PoE @ $50 \mathrm{~g}$ a.i./ha) treatment which was statistically equivalent. No significant difference was observed in fresh herbage yield in T1 (Pendi. PE @ 1 kg a.i./ha + MW at 30-35 DAT) and T2 (Quizalofop ethyl PoE @ 50 g a.i./ha + MW at 30-35 DAT) treatment. After weed free treatment, fresh herbage yield was significantly higher in T8 (Three hand weeding) treatment than all other treatments, except T1 (Pendi. PE @ $1 \mathrm{~kg}$ a.i./ha + MW at 30-35 DAT) and T4 (Pendi. PE @ 1 kg a.i./ha. + Quizalofop ethyl PoE @ $50 \mathrm{~g}$ a.i./ha $+\mathrm{MW}$ at 30-35 DAT) treatment which was statistically at par.

The maximum dry biomass yield (4.693 t/ha) was recorded in weed free treatment which was significantly higher than all other treatments. The lowest dry biomass yield (1.437 t/ha) was recorded in weedy check treatment which was significantly lower than all other treatments, except T3 (Pendi. PE @ $1 \mathrm{~kg}$ a.i./ha + Quizalofop ethyl PoE @ $50 \mathrm{~g}$ a.i./ha) and T2 (Quizalofop ethyl PoE @ $50 \mathrm{~g}$ a.i./ha + MW at 30-35 DAT) treatmentsimilar result was observed by Singh and Sirohi (1983).

Statistically no significant difference was observed in dry biomass yield in T5 (Pendi. PE@1 kg a.i./ha + straw mulch @3 t/ha) and T6 (Pendi. PE @ 1 kg a.i./ha + straw mulch @ 5 t/ha) treatment ZaniewiczBajkowska et al.,(2009).

Maximum dry and fresh herbage yield was observed in weed free treatment. It was mainly due to effective control of all weeds in weed free treatment by repeated hand weedings at various stages of crop growth which significantly increased dry and fresh biomass yield. Similar results were also obtained by Gupta et al., (2014).

The lowest dry and fresh herbage yield was recorded in weedy check treatment. It was mainly due to uncontrolled weeds situation resulted heavy infestation of weeds which suppressed of crop plants and decreased fresh and dry biomass yield. 
Table.1 Treatment details of the experiment

\begin{tabular}{|c|c|c|}
\hline S.no. & Treatment & Treatment details \\
\hline 1. & $\mathrm{~T}_{1}$ & $\begin{array}{l}\text { Pendimethalin PE @ } 1 \mathrm{~kg} \text { a.i. ha }{ }^{-1} \text { followed by mechanical weeding by } \\
\text { hand hoe at 30-35 DAT }\end{array}$ \\
\hline 2. & $\mathrm{~T}_{2}$ & $\begin{array}{l}\text { Quizalofop ethyl PoE at 3-5 leaf stage of weeds @ } 50 \mathrm{~g} \text { a.i. ha }{ }^{-1} \\
\text { followed by mechanical weeding by hand hoe at 30-35 DAT }\end{array}$ \\
\hline 3. & $\mathrm{~T}_{3}$ & $\begin{array}{l}\text { Pendimethalin PE @ } 1 \text { kg a.i. ha }{ }^{-1}+\text { Quizalofop ethyl PoE at 3-5 leaf } \\
\text { stage of weeds @ } 50 \text { g a.i. ha }{ }^{-1}\end{array}$ \\
\hline 4. & $\mathrm{~T}_{4}$ & $\begin{array}{l}\text { Pendimethalin PE @ } 1 \mathrm{~kg} \text { a.i. ha }{ }^{-1}+\text { Quizalofop ethyl PoE at } 3-5 \text { leaf } \\
\text { stage of weeds @ } 50 \mathrm{~g} \text { a.i. ha }{ }^{-1} \text { followed by mechanical weeding by hand } \\
\text { hoe at } 30-35 \text { DAT }\end{array}$ \\
\hline 5. & $\mathrm{~T}_{5}$ & Pendimethalin PE @ 1 kg a.i. ha ${ }^{-1}+$ straw mulch @ 3 t ha $^{-1}$ \\
\hline 6. & $\mathrm{~T}_{6}$ & Pendimethalin PE @ 1 kg a.i. ha ${ }^{-1}+$ straw mulch @ $5 \mathrm{t} \mathrm{ha}^{-1}$ \\
\hline 7. & $\mathrm{~T}_{7}$ & Two hand weedings at 15-20 and 30-35 DAT \\
\hline 8. & $\mathrm{~T}_{8}$ & Three hand weeding at $15-20,30-35$ and $45-50$ DAT \\
\hline 9. & $\mathrm{~T}_{9}$ & Weedy check \\
\hline 10. & $\mathrm{~T}_{10}$ & Weed free \\
\hline
\end{tabular}

$\mathrm{PE}=$ pre-emergence, $@=$ at the rate, a.i. = active ingredient, ha = hectare, DAT = days after transplanting, PoE = post-emergence, T1-Pendimethalin PE @ $1 \mathrm{~kg}$ a.i. ha-1 + mechanical weeding at 30-35 DAT, T2-Quizalofop ethyl PoE @ 50 g a.i. ha-1 + mechanical weeding at 30-35 DAT, T3-Pendimethalin PE @ 1 kga.i. ha-1 + Quizalofop ethyl PoE @ 50 g a.i. ha-1, T4-Pendimethalin PE @ 1kga.i. ha-1 + Quizalofop ethyl PoE@ 50 ga.i. ha-1 + mechanical weeding at 30-35 DAT, T5-Pendimethalin PE @ 1 kga.i. ha-1 + straw mulch @ 3 t ha-1, T6Pendimethalin PE @ 1 kg a.i. ha-1 + straw mulch @ 5 t ha-1, T7-Two hand weeding at 15-20 and 30-35 DAT, T8Three hand weeding at 15-20, 30-35 and 45-50 DAT, T9-Weedy check, T10-Weed free.

Table.2 Fresh and dry biomass yield (t/ha) as influenced by different weed management treatments at harvest

\begin{tabular}{|l|l|l|}
\hline \multicolumn{1}{|c|}{ Treatments } & \multicolumn{1}{|c|}{$\begin{array}{c}\text { Fresh biomass } \\
\text { yield }\end{array}$} & $\begin{array}{c}\text { Dry biomass } \\
\text { yield }\end{array}$ \\
\hline $\mathrm{T}_{1}$ : Pendi. PE @ 1 kg a.i.//ha + MW at 30-35 DAT & 15.813 & 2.823 \\
\hline $\mathrm{T}_{2}$ : Quiza. PoE @ 50 g a.i./ha + MW at 30-35 DAT & 14.940 & 2.000 \\
\hline $\mathrm{T}_{3}$ : Pendi. PE @ 1 kg a.i./ha + Quiza. PoE @ 50 g a.i./ha & 12.027 & 1.863 \\
\hline $\begin{array}{l}\mathrm{T}_{4}: \text { Pendi. PE @ 1 kg a.i./ha. + Quiza. PoE @ 50 g a.i./ha } \\
\text { + MW at 30-35 DAT }\end{array}$ & 17.163 & 3.307 \\
\hline $\mathrm{T}_{5}:$ Pendi. PE @ 1 kg a.i./ha + straw mulch @ 3 t/ha & 13.997 & 2.343 \\
\hline $\mathrm{T}_{6}:$ Pendi. PE @ 1 kg a.i./ha + straw mulch @ 5 t/ha & 14.217 & 2.627 \\
\hline $\mathrm{T}_{7}:$ Two HW at 15-20 \& 30-35 DAT & 15.347 & 2.343 \\
\hline $\mathrm{T}_{8}:$ Three HW at 15-20, 30-35 \& 45-50 DAT & 20.640 & 3.817 \\
\hline $\mathrm{T}_{9}:$ Weedy check & 7.547 & 1.437 \\
\hline $\mathrm{T}_{10}:$ Weed free & 25.070 & 4.693 \\
\hline $\mathrm{SE}(\mathrm{m}) \pm$ & 1.633 & 0.230 \\
\hline $\mathrm{CD}(\mathrm{P}=0.05)$ & 4.890 & 0.689 \\
\hline
\end{tabular}


Table.3 Cost of cultivation, gross return, net return and B: C ratio as influence by different weed management practices

\begin{tabular}{|c|c|c|c|c|}
\hline Treatments & $\begin{array}{c}\text { Cost of } \\
\text { cultivation } \\
\text { (//ha) }\end{array}$ & $\begin{array}{c}\text { Gross } \\
\text { return } \\
\text { ('/ha) }\end{array}$ & $\begin{array}{l}\text { Net return } \\
(\text { (/ha) }\end{array}$ & $\begin{array}{l}\text { B: } \mathrm{C} \\
\text { ratio }\end{array}$ \\
\hline $\begin{array}{l}\mathrm{T}_{1} \text { : Pendi. PE @ } 1 \mathrm{~kg} \text { a.i./ha }+\mathrm{MW} \text { at } 30-35 \\
\text { DAT }\end{array}$ & 42871 & 564600 & 521729 & 12.16 \\
\hline $\begin{array}{l}\mathrm{T}_{2} \text { : Quiza. PoE @ } 50 \mathrm{~g} \text { a.i./ha }+\mathrm{MW} \text { at } 30-35 \\
\text { DAT }\end{array}$ & 43303 & 400000 & 356697 & 8.23 \\
\hline $\begin{array}{l}\mathrm{T}_{3}: \text { Pendi. PE @ } 1 \text { kg a.i./ha + Quiza. PoE @ } \\
50 \text { g a.i./ha }\end{array}$ & 41151 & 372600 & 331449 & 8.05 \\
\hline $\begin{array}{l}\text { T4: Pendi. PE @ } 1 \mathrm{~kg} \text { a.i./ha. + Quiza. PoE @ } \\
50 \mathrm{~g} \text { a.i./ha + MW at } 30-35 \text { DAT }\end{array}$ & 45291 & 661400 & 616109 & 13.60 \\
\hline $\begin{array}{l}\mathrm{T}_{5}: \text { Pendi. PE @ } 1 \text { kg a.i./ha + straw mulch @ } \\
3 \mathrm{t} / \mathrm{ha}\end{array}$ & 40151 & 468600 & 428449 & 10.67 \\
\hline $\begin{array}{l}\mathrm{T}_{6}: \text { Pendi. PE @ } 1 \text { kg a.i./ha + straw mulch @ } \\
5 \mathrm{t} / \mathrm{ha}\end{array}$ & 40791 & 525400 & 484609 & 11.88 \\
\hline $\mathrm{T}_{7}:$ Two HW at $15-20 \& 30-35$ DAT & 45483 & 468400 & 422917 & 9.29 \\
\hline $\mathrm{T}_{8}$ : Three HW at $15-20,30-35 \& 45-50$ DAT & 49623 & 763400 & 713777 & 14.38 \\
\hline $\mathrm{T}_{9}:$ Weedy check & 37203 & 287400 & 250197 & 6.72 \\
\hline $\mathrm{T}_{10}:$ Weed free & 57903 & 938600 & 880697 & 15.20 \\
\hline $\mathrm{SE}(\mathrm{m}) \pm$ & - & $46,076.74$ & $46,076.74$ & 0.883 \\
\hline $\mathrm{CD}(\mathrm{P}=0.05)$ & - & 137961 & 137961 & 2.643 \\
\hline
\end{tabular}

Fig.1 Fresh and dry biomass yield (t/ha) as influenced by different weed management treatments at harvest

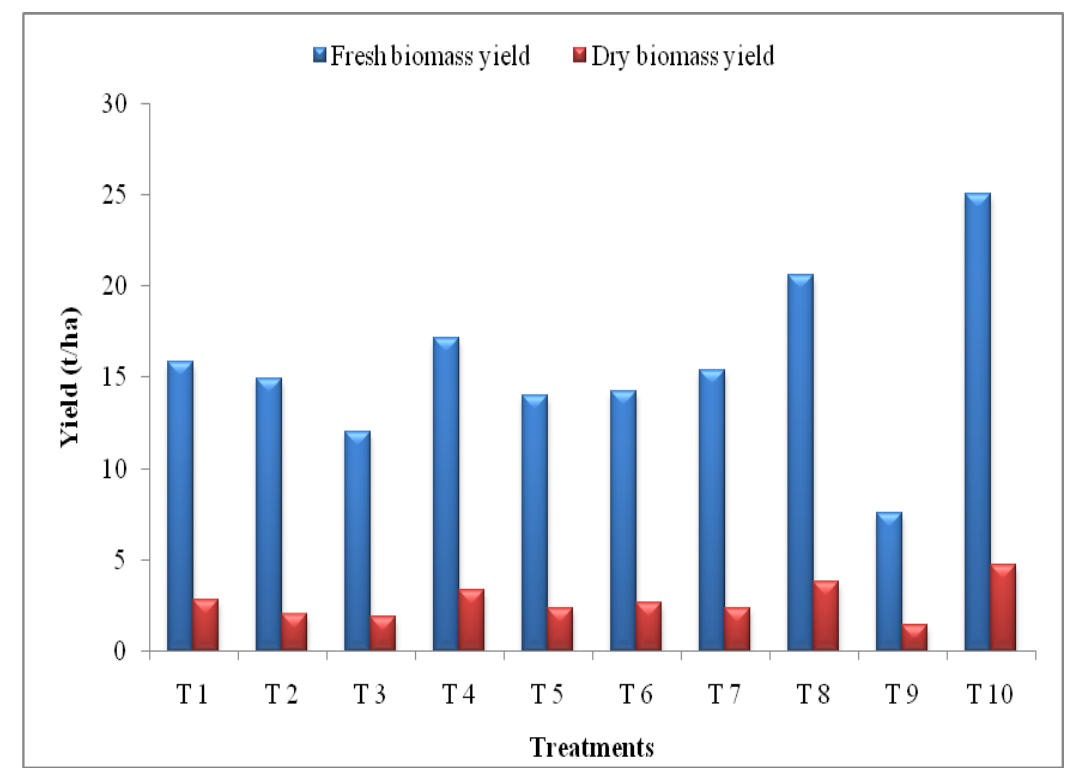




\section{Economics}

The data on economic studies of different weed control treatments are presented in Table 3.2.The data on economic studies revealed that the maximum net return were obtained under weed free treatment followed by $\mathrm{T} 8$ (Three hand weeding), T4 (Pendi. PE @ 1 kg a.i./ha. + Quizalofop ethyl PoE @ $50 \mathrm{~g}$ a.i./ha + MW) and T1 (Pendi. PE @ $1 \mathrm{~kg}$ a.i./ha + MW at 3035 DAT) treatment. The minimum net return was obtained in weedy check treatment.

The data on benefit cost ratio are presented in Table 3.2. The B: $\mathrm{C}$ ratio (15.20) under weed free treatment was significantly higher than all other treatments, except T8 (Three hand weeding) and T4 (Pendi. PE @ 1 kg a.i./ha. + Quizalofop ethyl PoE @ $50 \mathrm{~g}$ a.i./ha + MW) treatments which was statistically equivalent. The B: C ratio (6.72) in weedy check treatment was significantly lower than all other treatments, except T2 (Quizalofop ethyl PoE @ $50 \mathrm{~g}$ a.i./ha + MW at 30-35 DAT), T3 (Pendi. PE@ 1 kg a.i./ha + Quizalofop ethyl PoE @ 50 $\mathrm{g}$ a.i./ha) and T7 (Two hand weeding) treatments.

It is concluded on the basis of experimental results, it could be concluded that the treatment T4 (Pendimethalin PE @ 1 kg a.i./ha. + Quizalofop ethyl PoE @ $50 \mathrm{~g}$ a.i./ha followed by mechanical weeding) may be taken as an alternative to the hand weeding for efficient weed control and achieving high biomass yield of kalmegh during kharif season.

\section{References}

Bonasia, A., Conversa, G., Lazzizera, C., La
Ratonda, P. and Elia, A. 2012. Weed control in lampascione (Muscaricomosum L.). Crop Protec., 36(2): $65-72$.

Chandraker, A.K. and Paikra, P.R. 2015. Effect of integrated weed management on weed dynamics of soybean [Glycine max (L.)Merrill] under Chhattisgarh plain. Indian J. Agric. Res., 49(1): 53-58.

Chaudhary, G., Goyal, S., and Poonia, P. 2010. "Lawsoniainermis Linnaeus: a phytopharmacological review," Int. J. Pharmaceutical Sci. Drug Res., 2(2): 9198.

Gupta, V., Singh, M., Kumar, A., Sharma, B.C. and Kher, D. 2014. Effect of different weed management practices in urdbean (Vigna mungo) under sub-tropical rainfed condition. Legume Res., 37(4): 424-429.

Joy, P., Thomas, J., Mathew, S., and Skaria, B.P. 1989. "Medicinal plants," Trop. Horticulture, 2: 449-632.

Mirjha, P.R., Prasad, S.K., Singh, M.K., Paikra, R.H., Patel, S. and Majumdar, M. 2013. Effect of weed control measures on weeds, nodulation growth and yield of mung bean (Vigna radiata). Indian $J$. Agron., 58(4): 615-617.

Singh, O.S. and Sirohi, S.S. 1983. Relationship of endomycorrhizal association of unsterilized soils with available soil phosphorus, plant growth, phosphorus uptake and oil synthesis in pepper mint. Scientia Horticulturae, 20(2): 185-191.

Zaniewicz-Bajkowska, A., Franczuk, J. and Kosterna, E. 2009.Direct and secondary effects of soil mulching with straw on fresh mass and number of weeds vegetable yield. Polish J. Environ. Stud., 18(6): 1185-1190.

\section{How to cite this article:}

Bolta Ram Meena, Shiv Singh Meena and Kala D.C. 2017. Assessment of Yield and Economic for Kalmegh (Andrographis paniculata Nees) by Using Different Weed Control Techniques. Int.J.Curr.Microbiol.App.Sci. 6(5): 2303-2308. doi: https://doi.org/10.20546/ijcmas.2017.605.257 